1 Calcium-Oxygen Batteries as a Promising Alternative to Sodium-Oxygen

4 Philip Reinsberg, Christoph J. Bondue and Helmut Baltruschat ${ }^{*}$

5

$\frac{6}{7}$

Institut für Physikalische und Theoretische Chemie, Universität Bonn, Römerstraße 164, D-53117

Bonn, Germany

8

$9 \quad{ }^{*}$ Corresponding author: baltruschat@uni-bonn.de

10 Supplementary Information

\title{
11 Experimental
}

12 Chemical reagents. Calcium perchlorate tetrahydrate (99\%, Sigma Aldrich) was 13 repeatedly dried over an extended period of time $(8 \mathrm{~h})$ at $250^{\circ} \mathrm{C}$ under reduced 14 pressure $\left(10^{-2} \mathrm{mbar}\right)$ giving a white powder with a water content below $20 \mathrm{ppm}$ 15 (measured in MeCN). Tetrabutylammonium perchlorate ( $\geq 99 \%$, Sigma Aldrich) was used as received.

17 The electrolyte preparation was carried out under an Ar-atmosphere in an MBraun 18 glovebox. Extra dry DMSO (99.7\%, over molecular sieve, Acros Organics) was used 19 as received. The DEMS-measurements were performed using a custom made 20 mixture of $\mathrm{Ar}$ and $\mathrm{O}_{2} \quad\left(80: 20=\mathrm{Ar}: \mathrm{O}_{2}\right)$ obtained from Air Liquid. Oxygen 21 concentrations for the RRDE-measurements were adjusted by mixing highly pure 22 Argon (Air Liquid, $99.999 \%$ ) and highly pure oxygen (Air Liquid, 99.999 \%) using two 23 flowmeters (Krohne Duisburg). The accuracy of the flowmeters was verified by 24 measuring the volume expansion of the flowing gases with respect to time.

25 Rotating-Ring-Disc-Electrode (RRDE) measurements. The RRDE-measurements 26 were performed in a closed $\mathrm{H}$-cell. This $\mathrm{H}$-cell was purged with an $\mathrm{Ar}-\mathrm{O}_{2}$ mixture 
1 throughout the experiment to saturate with oxygen and avoid contamination of the

2 electrolyte with water from the ambient air. The water content was determined after

3 the experiment via Karl-Fischer-titration (approx. 40 ppm). A silver wire in a solution

4 of $0.1 \mathrm{M} \mathrm{AgNO}_{3}$ in $\mathrm{DMSO}$ was used as reference electrode. To avoid contamination

5 of the working electrolyte with $\mathrm{AgNO}_{3}$ the contact between reference electrode and

6 working compartment was established via the wet surface of a closed glass

7 stopcock. The geometric surface area of the glassy carbon-disc electrode was

$8 \quad A(\mathrm{GC})=0.196 \mathrm{~cm}^{2}$, whereas a different tip (thin-gap) with $A(\mathrm{Pt})=0.164 \mathrm{~cm}^{2}$ was used

9 as the Pt-electrode. The collection efficiency $N_{0}$ of the GC-disc and Pt-ring

10 arrangement was calculated to $N_{0}(\mathrm{GC}-\mathrm{Pt})=0.24$. The thin-gap RRDE-tip consisting

11 of a Pt-disc and -ring had a collection efficiency of $N_{0}(\mathrm{Pt}-\mathrm{Pt})=0.22$.

12 Differential electrochemical mass spectrometry (DEMS) measurements. Two

13 different cells were used in order to carry out the DEMS-measurements. The first cell,

14 the dual thin-layer cell, consists of two compartments. The electrochemical reaction

15 takes place in the upper compartment (working compartment), where the working

16 electrode is placed. The lower compartment (detection compartment), is connected

17 to a mass spectrometer (MS) via a porous Teflon membrane. Both compartments are

18 connected via six capillaries. The porous Teflon membrane rests on a steel frit and

19 allows volatile species to diffuse into the vacuum. However, the solvent itself cannot

20 pass the membrane due to its high surface tension and low vapour pressure. Due to

21 continuous flow, products formed in the upper compartment are transported to the

22 lower compartment where they may evaporate into the vacuum of the mass

23 spectrometer. The simultaneous acquisition of the faradaic current $/ F$ at the working

24 electrode and the ionic current related to the mass $z l_{z}$ allows a direct identification of 
1 (volatile) products and the calculation of the number of electrons transferred per 2 products molecule.

3 The second cell, the 6-electrode dual thin-layer cell, also consists of two 4 compartments. The major difference to the first cell is that a metal-sputtered 5 membrane can be employed as a detection electrode in the lower compartment. This 6 is not possible in the dual thin layer cell because the high ohmic drop inhibits 7 potential control of the detection electrode. This problem was circumvented by 8 employing two distinct sets of three electrode arrangements (one working electrode, 9 one reference electrode and one counter electrode). Each working electrode was 10 controlled by a potentiostat. The potentiostat of the detection electrode was 11 decoupled from ground. The six electrode cell is reminiscent of an RRDE12 arrangement: The metal sputtered Teflon membrane is held at a fixed potential at which species formed at the working electrode in the upper compartment can undergo an electrochemical reaction. The advantage of the 6-electrode cell over a conventional RRDE-arrangement is the option to combine a detection electrode with mass spectroscopy. It is, therefore, possible to detect the products of the electrochemical reaction at the working electrode in the lower compartment as well as the products of the electrochemical reaction at the working electrode in the upper compartment.

For both cells a reference electrode consisting of a silver wire in $\mathrm{Ag} / \mathrm{AgNO}_{3}$ in 21 DMSO was connected via a Teflon-tube, which was closed by a rough glass bead, to 22 the cell. The ionic current related to mass $32 l_{32}$ is considered to be the ionic current 23 due to $\mathrm{O}_{2}$, while $I_{44}$ should resemble the ionic current due to $\mathrm{CO}_{2}$. Especially the first 24 assumption was further assured by introducing oxygen into the electrolyte and 25 observing the change of $/ 32$. All ionic currents presented here have been subject to a 
1 background subtraction and a smoothing process. The geometric surface areas of

2 the working electrodes in the upper compartment amounted to $A=0.283 \mathrm{~cm}^{2}$.

3 Calibration of the dual thin-layer cell. The number of electrons $z$ transferred per

4 oxygen molecule can be calculated according to equation (1) as the ionic current $l_{32}$

5 (which is directly measurable) is proportional to the amount of oxygen entering the

6 MS per time and the faradaic current $I_{F}$ is proportional to the amount of oxygen,

7 which is converted at the electrode, times the number of transferred electrons:

$$
z=\frac{I_{F}}{I_{32}} \cdot K^{*}=\frac{I_{F}}{\dot{n}\left(\mathrm{O}_{2}\right) \cdot F}
$$

8 To alleviate understanding of the data presented in the main paper, the flow of

9 oxygen $\dot{n}\left(\mathrm{O}_{2}\right)$ is plotted in the figures. The mathematical relation between $\dot{n}\left(\mathrm{O}_{2}\right)$ and $10 \quad l_{32}$ is obvious from equation (1) where $F$ denotes Faraday's constant.

11 In order to determine the calibration constant $K^{*}$ calibration of the system with a 12 reaction of known stoichiometry (known $z$ ) is required. For that purpose, the ORR in 13 the presence of $\mathrm{TBAClO}_{4}$ was chosen, which was already reported to yield 14 superoxide quantitatively. $K^{*}$ does not only contain all the settings of the mass 15 spectrometer that determine its sensitivity but also the probability that a molecule 16 enters the vacuum in the first place. Therefore, $K^{*}$ is only the proportionality constant 17 that relates the faradaic current at the working electrode in the upper compartment to 18 the corresponding ionic current. Thus, if $z$ is known, equation (1) can be used to 19 determine $K^{*}$.

20 Calibration of the six-electrode dual thin-layer cell. In order to relate the faradaic 21 current at the detection electrode WE2 to the corresponding ionic current a second 22 calibration constant $K_{2}{ }^{*}$ is required. $K_{2}{ }^{*}$ will differ from $K^{*}$ because the probability of a 
1 volatile product to enter the vacuum is larger. That is due to the fact that the

2 detection electrode is placed at the interface between vacuum and electrolyte.

3 The measurement used for calibration of the six-electrode dual thin-layer cell is

4 shown in Fehler! Verweisquelle konnte nicht gefunden werden.. $K_{2}{ }^{*}$ as well as $K^{*}$

5 is determined by the reduction of oxygen to superoxide in TBA+-containing DMSO. In

6 the detection compartment superoxide is re-evolved to oxygen. This is a problem

7 because the MS-signals of both working electrodes overlap: Oxygen is consumed at

8 the working electrode in the upper compartment and oxygen is formed at the

9 detection electrode. Hence, we can only determine the sum of the ionic current

$10 \sum I_{32}$ of both processes. However, in order to determine $K_{2}{ }^{*}$ we require the ionic

11 current $l_{32, \text { WE2 }}$ due to the reaction at the detection electrode WE2. The ionic current $l_{32}$

12 due to the reaction at the working electrode WE1 in the upper compartment equals

$13 \sum I_{32}$ when no reaction takes place in the lower compartment. Therefore, $I_{32, \text { WE2 } 2 \text { can }}$

14 be determined when $I_{32}$ (no reaction in the lower compartment) is subtracted from the

$15 \sum \sum l_{32}$ (detection reaction in the lower compartment). 


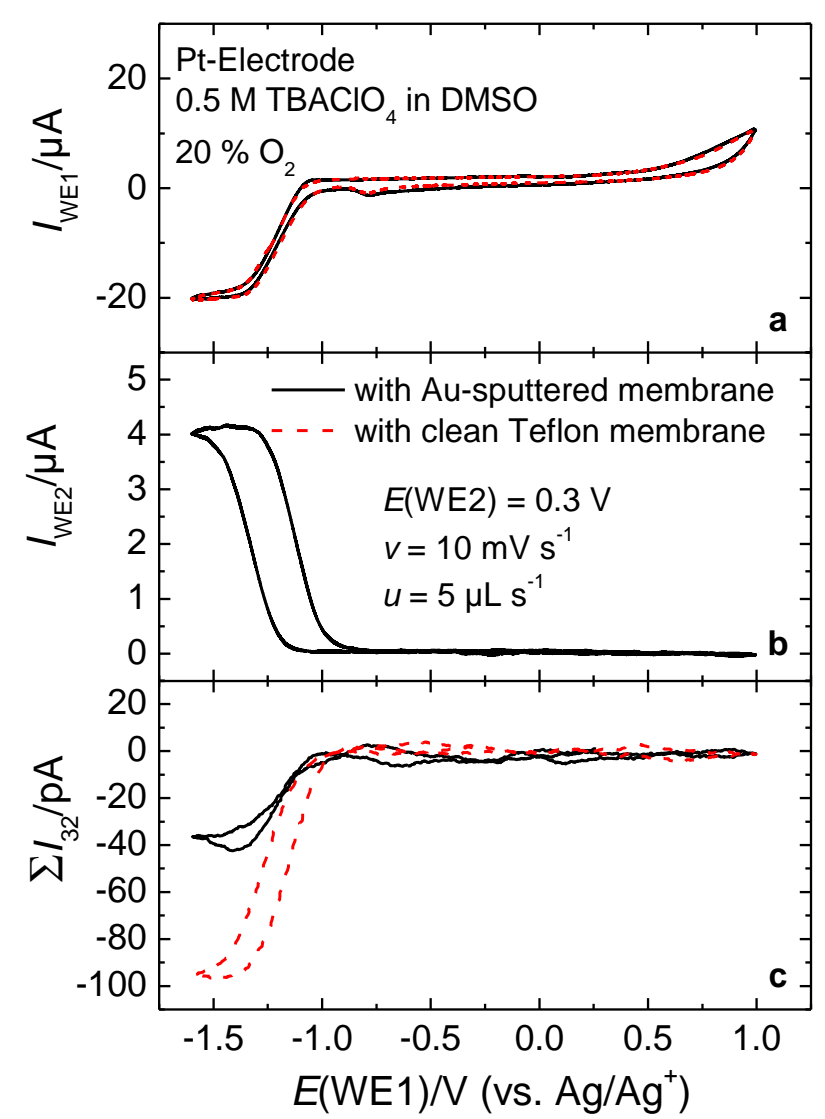

2 Figure S1: Calibration of the six-electrode dual thin-layer cell at a Pt-electrode. a, CVs at the 3 working electrode WE1 in the upper compartment. $\mathbf{b}$, Currents at the porous Au-electrode in the lower 4 compartment (WE2) at a constant potential of $E(W E 2)=0.3 \mathrm{~V}$. c, Corresponding ionic currents 5 measured. Electrolyte: $0.5 \mathrm{M} \mathrm{TBAClO}_{4}$ in DMSO saturated with $20 \% \mathrm{O}_{2}$ and $80 \% \mathrm{Ar}$.

$6 K_{2}{ }^{*}$ is then determined via equation (2). It is also possible to determine the number of 7 electrons that are transferred during the reaction at the detection electrode via 8 equation (2) if $K_{2}{ }^{*}$ is known. Hence, allowing the experimentalist to gather some 9 information on the nature of the intermediate that reacts at the detection electrode.

$$
K_{2}^{*}=\frac{I_{32, \mathrm{WE} 2}}{I_{\text {WE2 }}}=\frac{\sum I_{32}-I_{32}}{I_{\text {WE2 }}}
$$

10 It might be interesting to evaluate the amount of species, which react at the 11 second working electrode with respect to the total amount of species generated at 12 WE1 (i.e. the share of superoxide $x$ in this case). This can be determined from the 
1 ratio of the current at the detection electrode IWE2 to the current /WE1 at the working

2 electrode in the upper compartment. This means that the transfer efficiency (the

3 analogue of the collection efficiency of an RRDE arrangement) is needed. In general,

4 there are also different methods to evaluate the share of superoxide. However, in this

5 publication we shall limit ourselves to the faradaic currents at WE1 and WE2 in order

6 to alleviate the comparability between RRDE and DEMS-experiments. In the case of

7 an RRDE, the maximum share of species, first produced at the disc-electrode and

8 then reacting at the ring-electrode, is defined by the theoretical collection efficiency

9 No, which is defined by the geometry of the arrangement. The theoretical collection

10 efficiency can also be measured: In such experiments a reversible redox system is

11 employed and potentials for the disc and ring-electrode are chosen such that the

12 reactions at both electrodes are limited by diffusion. The collection efficiency is then

13 determined from the ratio of the current at the ring electrode to the current at the disc

14 electrode.

15 Similar to this, a calibration constant which relies on the faradaic currents at WE1

16 and WE2 can be defined for the 6-electrode dual thin-layer cell. This calibration

17 constant $N_{0} \mathrm{~F}$ (the faradaic transfer efficiency) also represents the maximum share of

18 products that can undergo a reaction at the detection electrode. $N_{0} \mathrm{~F}$ depends on the

19 geometry of the cell, the flow rate $u$ and the diffusion coefficient of the species under

20 investigation. According to equation (3), $N_{0} F$ can be determined from the results

21 presented in Fig. S 1.

$$
N_{0}^{F}=\left|\frac{I_{W E 2}}{I_{W E 1}}\right|
$$

22 The calibration constant $N_{0} \mathrm{~F}$ can now be used to calculate the share of superoxide 23 on the total amount of formed species in a manner, which is analogous to the 
1 evaluation of RRDE-measurements. If the assumption is made that either superoxide

2 or some small amount of peroxide is formed at the first working electrode WE1, the

3 total current observed at WE1 can be written as:

$$
I_{W E 1}=(2-x) \cdot F \cdot\left(\frac{d n}{d t}\right)
$$

$4 \quad \mathrm{x}$ in equation (4) represents the share of superoxide, $F$ is the Faradaic constant 5 and $d n / d t$ is the number of moles reacting per unit of time. At the same time, the

6 current observed at the second working electrode can be written as

$$
I_{\text {WE2 }}=Z \cdot F \cdot N_{0}^{F} \cdot\left(\frac{d n}{d t}\right)
$$

7 By assuming that only superoxide is oxidised at the ring (which is indeed justified as

8 Fig. 2 shows), combination and rearrangement of equation (4) and (5) leads to:

$$
x=\frac{2 \cdot I_{\text {WE2 }}}{I_{\text {WE1 }} \cdot N_{0}^{F}+I_{\text {WE2 }}}
$$

9 Equation (6) resembles the equation used to calculate the share of intermediates for

10 RRDE assemblies.

11 Tafel-plots at the GC-electrode. The Tafel-plot in Figure S2 indicates, in 12 accordance with previous measurements, that a one-electron transfer is the rate 13 determining step for the ORR at glassy carbon. 


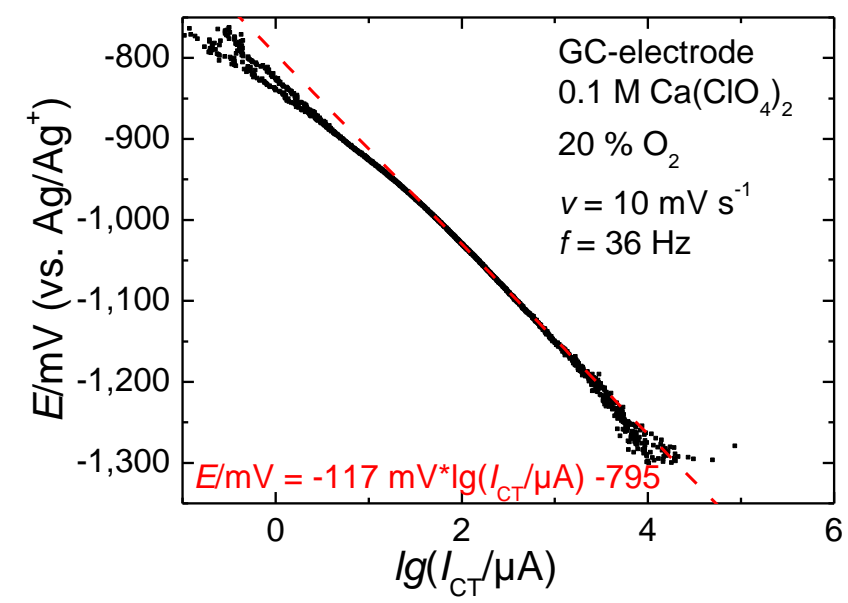

2 Figure S2: Tafel-plot using the data of Fig. 4. $I_{\mathrm{CT}}$ indicates the current due to the charge-transfer 3 without any kind of diffusion overpotential.

4 Comparison of RRDE experiments at GC- and Pt-electrodes. As already stated in 5 the main section, it is expected from the electron numbers (Fig. 1d) that the ORR at 6 GC- and Pt-electrodes is rather similar. To further compare the ORR at GC- and Pt7 electrodes, RRDE measurements are presented in Figure S 3.
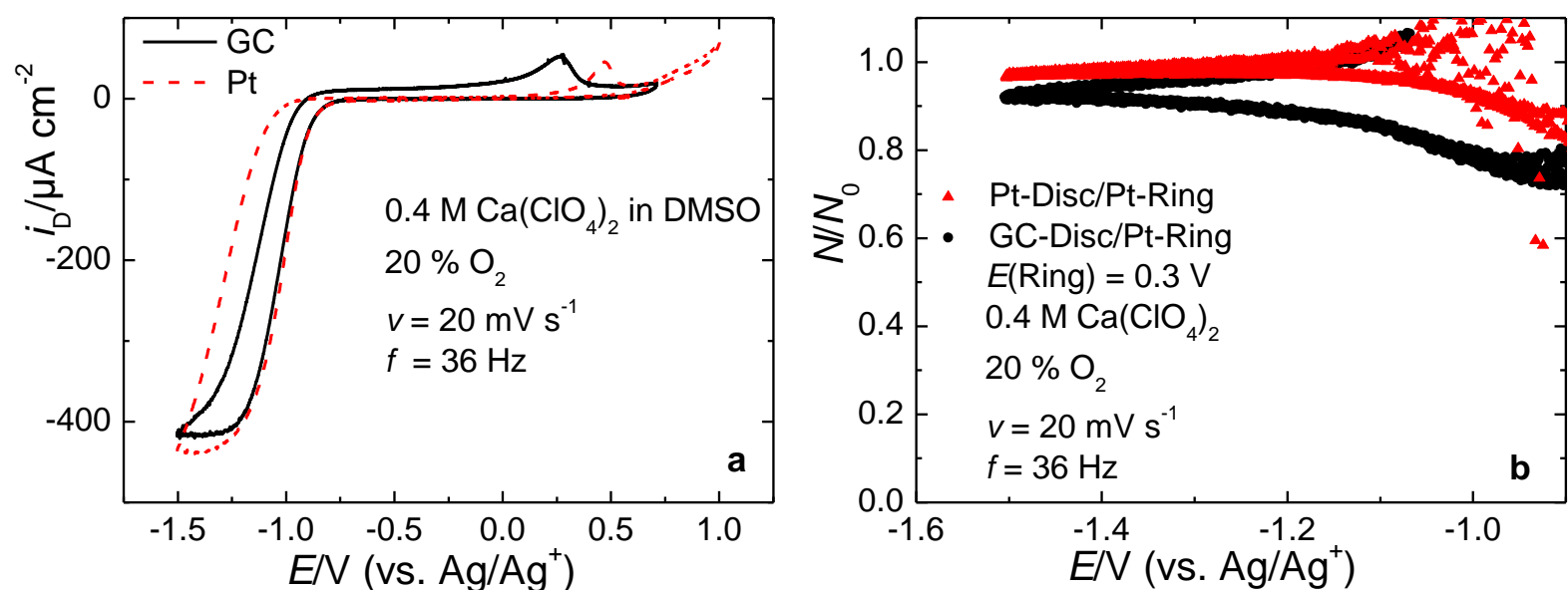

8 Figure S 3: Comparison of the RRDE-measurements at GC- and Pt-discs. a, Comparison of the

9 disc-currents at a GC (black)- and Pt-electrode with respect to the geometric surface area. b, 10 Comparison of the collection efficiency $N$ with respect to the theoretical collection efficiency $N_{0}$ at a 11 ring-potential of $0.3 \mathrm{~V}$.

12 A Pt ring-electrode was used in each of these different arrangements. Instead of 13 the measured disc-currents the disc-currents with respect to the geometric surface 14 are used, as the Pt-electrode was a preassembled thin-gap electrode with a different 15 geometric area (and a different theoretical collection efficiency of $N_{0}=0.22$ ). 
1 Comparing the diffusion-limited currents between $-1.3 \mathrm{~V}$ and $-1.5 \mathrm{~V}$, the electrodes

2 behave rather similar. Deviations can mainly be attributed to slightly different

3 concentrations of oxygen. The difference in the collection efficiency might be

4 explained by the fact that the Pt-Pt-arrangement is preassembled and thus might be

5 in better agreement with the theoretical value of the collection efficiency than the GC-

6 Pt-arrangement, which is a change-disc assembly.

7 A difference between GC on the one hand and $\mathrm{Pt}$ on the other hand is the 8 hysteresis between anodic and cathodic sweep, which is more pronounced in the 9 case of $\mathrm{Pt}$ (Fig. S 3a). This is not unexpected regarding the generally weak 10 adsorption observed on GC-electrodes. The anodic peak at the Pt-electrode is

11 shifted into the positive direction, which is in agreement with the larger hysteresis 12 observed. To sum up, GC appears to be the more promising electrode material as 13 compared to Pt. 